Orte gemachten Mitteilungen immer mehr an Wahrscheinlichkeit gewinnt, daß das hochwertige Meningococcenserum tatsächlich, wenn frühzeitig angewandt, ein Heilserum für diese Krankheit bei Verwendung genügend großer Mengen darstellt, erhalten die rein wissenschaftlichen Untersuchungen über den Gehalt des Serums an spezifischen Stoffen mehr und mehr Bedeutung.

Kolle und Was serman n hatten bekanntlich vorgeschlagen, die Wertbestimmung des Meningococcenserums mittels der sogenannten Komplementverankerungsmethode nach Bordet. G'engou vorzunehmen. Der Grund für die Wahl dieser Methode war vor allem darin zu suchen, daß man bei der Prüfung des Meningococcenserums nicht allein auf die Agglutinine bzw. Präzipitine, welche einen Rückschluß auf den Gehalt an Schutzstoffen nicht ohne weiteres erlauben, angewiesen war.

Agglutinine werden im Körper der für die Zwecke der Serumlieferung immunisierten Tiere in steigender Menge gebildet; erfolgt die Immunisierung kunstgerecht und werden steigende Dosen der Meningococcenleiber intravenös injiziert, so geht der Titer der Agglutination der Menge der injizierten Coccenleiber annähernd parallel. Diese Meningococcenagglutinine sind durchaus spezifisch; nicht nur unsere eigenen Untersuchungen sowie die von Kutscher, ${ }^{1}$ ) Vannod, ${ }^{2}$ ) Lingelsheim, ${ }^{3}$ ) Fischer unter Bonhoffs Leitung ${ }^{4}$ ) u. a. unter Zuziehung zahlreicher Coccenarten ausgeführten Untersuchungen, sondern auch die jüngst erschienenen Arbeiten von Ballner und Reibmeyer ${ }^{5}$ ) haben das hinreichend sichergestellt. Wenn neuerdings Eberle ${ }^{6}$ ) in einer unter Silberschmidts Leitung ausgeführten Arbeit die Agglutinationsreaktion weder für die Identifizierung der Meningococcen noch für die Differenzierung ähnlicher Mikroorganìsmen als ausschlaggebend betrachtet und ihr überhaupt keinen großen praktischen Wert zuerkennt, so befindet er sich in einer ganz isolierten Stellung. Wir müssen seine Versuchsergebnisse unberücksichtigt lassen, da unsere zahlreichen, fast täglich ausgeführten Untersuchungen stets wieder das Gegenteil von dem beweisen, was Eberle behauptet. Als Beispiel für die Spezifizität der Agglutination hochwertigen Meningococcenserums geben wir die folgende kleine Tabelle wieder. Es ist bei

$$
\text { Tabelle I. }
$$

Agglutinationsprobe mit Berner Meningococcenserum gegen Meningococcenstamm Beuthen, Berlin, Biel, Moutier, III., Gonococcen und Micrococcus catarrhalis.

Aus aem institut zur Ertorschung der Intektionskrankheiten in Bern. (Direktor: Prof. Dr. W. Kolle.)

\section{Untersuchungen über das Meningococcen- serum.}

Von Dr. Krumbein, technischem Leiter des Schweizerischen Serum- und Impfinstituts, und Dr. P. Schatiloff, Privatdozent an der Universität Charkow.

Nachdem es durch die neueren klinischen Beobachtungen, namentlich von Schöne, $\left.{ }^{2}\right)$ Levy,,$\left.^{3}\right)$ Robb,4) und die von Wassermann ${ }^{5}$ ) auf dem Internationalen Kongreß für Hygiene und Demographie in Berlin, sowie von $\mathrm{Kolle}^{6}$ ) am gleichen

1) Zeitschrift fär klinische Medizin 1898, Bd. 35, H. 5-6

2) O. Schö ne, Inauguraldissertation. Darmstadt 1906. - 3) Lev y, Klinisches Jahrbuch 1908, Bd. 18. Deutsche medizinische Wochenschrift 1908, No. 4. - 4) Rob b Jahrbuch 1908, Bd. 18. Deutsche medizinische Wochenschrift 1908, No. 4. - 4) R ob b British Medical Journal. 15. Februar 1908. - 5 ) Wassermann, Deutsche medizinische Wochenschrift No. 39. - 6) Kolle, Verhandlungen des XIV. Internationalen
Kongresses für Hygiene und Demographie. 
Erfahrungen, auf welche weiter unten zurückzukommen sein wird.

Die Herbeiziehung des Tierversuchs zur Prüfung des Meningococcenserums auf seine antiinfektiösen Eigenschaften und die etwaige Heilkraft ist seitens Ruppels bereits benutzt. Leider ist die von Ruppel gezüchtete Meningococcenkultur keinem Bakteriologen zur Nachprüfung zugänglich geworden; sie soll in einer Dosis von ${ }_{100000}^{1}$ Oese bei intraperitonealer Einverleibung weiße Mäuse in 24 Stunden töten. Es ist uns zwar auch gelungen, einzelne Meningococcenstämme durch länger dauernde Züchtungen auf flüssigen Nährmedien, denen natives Eiweiß und Blut (Menschen- oder Tierblut) zugesetzt war, so zu verändern, da0 sie Mäuse in kleinen Mengen ( $1 / 20$ Oese) töteten. Hierüber hat Prof. Kolle auf dem Internationalen Kongre $B$ in Berlin bereits berichtet. Aber die lange Zeit fortgesetzten Versuche haben ergeben, daß es doch nich gelingt, gleichmäßigo Resultate zu erzielen. Die Virulenz der Kulturen läßt sich nicht auf die Dauer erhöhen, sie bleibt schwankend, und die dosis certe letalis läßt sich auch nicht über $1 / 20$ Oese pro Maus von $20 \mathrm{~g}$ Gewicht bei intraperitonealer Einverleibung steigern; einzelne der so für Mäuse virulent gemachten Kulturen haben ihre Virulenz später wieder eingebüßt, ohne daß es gelang, den Grund für dieses Verhalten herauszufinden. Auch alle andern Verfahren, die Infektiosität der Meningococcen für Mäuse oder Meerschweinchen zu erhöhen, sind trotz unserer vielfachen Bemühungen, ebenso wie die von den früheren Experimentatoren v. Lingelsheim, Leuchs ${ }^{1}$ ), Kutscher ${ }^{2}$ ) u. a. nicht erfolgreich gewesen. Wenn neuerdings Flexner und Jobling ${ }^{3}$ ) bei Affen die Erzeugung einer Meningitis in einigen Versuchen gelungen ist, so bedarf es doch noch weiterer Studien, um zu beweisen, daß dies regelmäßig gelingt. Für die Ausarbeitung einer Wertbestimmungsmethode des Meningococcenserums kommen Affen aber schon aus äußeren Gründen nicht in Frage.

Aus allen diesen Gründen ist es notwendig, die von Kolle und Wassermann angegebene Methode der Wertbestimmung des Meningococcenserums mittels der Komplementverankerung nach Bordet-Gengou bis auf weiteres beizubehalten; gleichzeitig müssen die Agglutinationstiter des Serums festgestellt werden.

Wir haben deshalb auf Veranlassung von Herrn Professor Kolle, dem alleVersuche unterbreitet worden sind, diese Methode eingehend experimentell weiter studiert. Es sei hier ausdrücklich vorweg bemerkt, daß die Frage offen gelassen werden soll, ob das Komplementverankerungsphänomen dem Ambozeptorengehalt eines Serums vollkommen parallel geht: das ist eine rein theoretische Frage. Der Kernpunkt liegt vielmehr in folgender Fragestellung: Leisten die mittels der Komplementverankerungsmethode als hochwertig festgestellten Sera auch therapeutisch beim Menschen etwas? Diese Frage ist gerade durch die klinischen Erfahrungen als im bejahenden Sinne beantwortet zu betrachten. Es ist deshalb nicht gerechtfertigt, wenn Kraus neuerdings dieses Verfahren als Prüfungsart für kurative Sera rundweg überhaupt und für das Meningococcenserum im besonderen ablehnt. Kraus und Dö $\mathrm{rr}^{4}$ ) hätten sich hier wohl etwas vorsichtiger ausdrücken können, um so mehr als die von ihnen ermittelten antitoxischen Wirkungen des Meningococcenserums und die darauf aufgebaute Wertbestimmungsmethode doch erst weiterer Bestätigungen und Erfahrungen bedürfen. In Kürze werden Krumbein und Dieh über die im schweizerischen Serum- und Impfinstitut angestellten Untersuchungen über Meningococcentoxine und den Gehalt des Serums an Antitoxinen berichten. Selbst wenn der Gehalt des Meningococcenserums an Antitoxinen zu einer Wertbe stimmung brauchbar sein sollte, würde damit der Wert der Komplementverankerungsprobe doch bestehen bleiben, weil die komplementverankernden Stoffe höchstwahrscheinlich besondere Körper der Immunsera sind.

Die Spezifizität der Komplementverankerung des Meningococcenserums ist eine durchaus strenge. Wie unsere mit

1) Klinisches Jahrbuch - 2) Klinisches Jahrbuch - 3) Journal of experimental medicine 1908 . Vol. X. - 4) Wiener klinische Wochenschrift 1908, No. 1, S. 12
Meningococcenserum und Meningococcen sowio ähnlichen Coccenarten istreptococcen, Staphylococcen, Gonococcen, Mikrococcus catarrhalis und andern Gram-negativen Coccen) angestellten L'ntersuchungen zeigen. gibt die Komplementverankerungsmethode sogar noch viel schärfere spezifische Resultate als die Agglutinationsreaktion.

Die folgende Tabelle zeigt, daß das Meningococcenserum bei Benutzung dieser Methode nur Meningococcen, nicht dagegen die andern Bakterienarten beeinflußt, und umgekehrt werden Meningococcen nicht durch andere Serumarten, z. B. Streptococcenserum, Gonococcenserum, Choleraserum mit dem Komplement verankert (Tabelle II.)

\section{Tabelle II a.}

Komplementverankerungsversuch mit Meningococcenserum Bern gegen Meningococcen, Staphylococcen, Gonococcen, Micrococcus catarrhalis und Streptococcen Methode Ne ufeld: Verwendetes Kulturquantum: 1/s Oese. Titer des hämolytischen Serums: 0,04 (inaktiv. bei $55^{\circ} 35$ Min.). Hammelblutkörper: Frisch, zweimal gewaschen und zentrifugiert. Komplement: Frisches Meerschweinchenserum 0,05. Meningococcenserum: Inaktiviert 1 Stunde bei $58-60^{\circ} \mathrm{C}$. Kochsalzlösung: Isotonisch $(0,85 \%) . \quad 7$ Kontrollen in variierter Zusammensetzung.

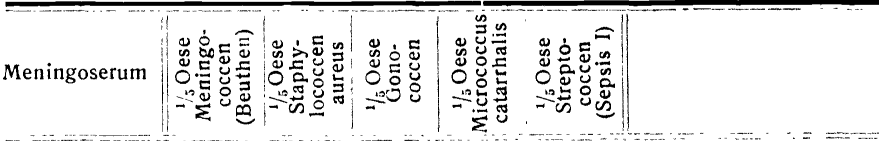

$$
\begin{aligned}
& \begin{array}{l|c|ll|l|l||l}
0,01 & \pm & 0 & 0 & 0 & 0 \\
0,007 & \pm & 0 & 0 & 0 & 0 \\
0,004 & ++0 & 0 & 0 & 0 & 0 \\
0,001 & 0 & 0 & 0 & 0 & 0 \\
00007 & 0 & 0 & 0 & 0 & 0
\end{array} \quad \mid \begin{array}{c}
7 \text { Kontrollen in Ord } \\
\text { nung. Beobachtung } \\
\text { nach i u. 24 Stunden. } \\
\text { Brutschrank. }
\end{array}
\end{aligned}
$$

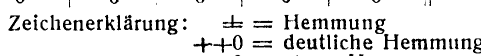

$$
\begin{aligned}
& ++0=\text { deutliche Hemm } \\
& .0=\text { Spur Hemmung }
\end{aligned}
$$$$
\text { Tabelle IIb. }
$$

Komplementverankerungsversuch mit Streptococcen-Gonococcen-Meningococcen-Cholera-Serum gegen Meningococcen: Hohe Serumdosen für die nicht spez. Sera, Versuchsanordnung wie oben.

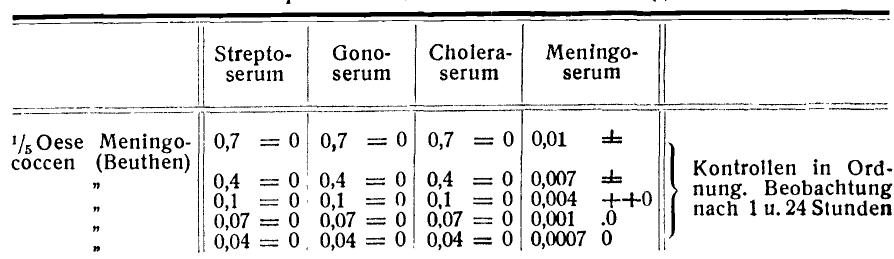

Diese Spezifizität zeigt sich nicht nur bei Benutzung der von Wassermann vorgeschlagenen Versuchsanordnung, bei welcher das Antigen in Form von Extrakten benutzt wird, sondern auch dann, wenn die ursprünglich von Bordet und Gengou angewandte Methodik benutzt wird. Neufeld ${ }^{1}$ ) und $\mathrm{Händel}{ }^{2}$ ) haben die Bordet-Gengousche Methode neuerdings bei Studien über das Choleraserum mittels der Komplementverankerung dadurch noch weiter modifiziert, daß sie die Bakterien, nachdem Serum und Komplement eine Stunde mit ihnen bei $37^{\circ}$ in Berührung gewesen waren, abzentrifugierten und nun die klare, bakterienfreie Flüssigkeit mit dem hämolytischen System versetzten. Es soll so die etwaige Lockerung des Komplements, wodurch nachträgliche Hämolyse eintritt, verhindert werden. Zum Vergleich der drei Varianten der Methode untereinander wurden nun eingehende Untersuchungen angestellt. Die Versuchsanordnung war dabei stets eine zweifache. In der einen Reihe wurde das Antigen in konstanten Mengen $(0,1)$ zugesetzt und das Meningococcenserum in fallenden Dosen, von $0,7-0,001 \mathrm{ccm}$; bei der zweiten Reihe wurde jedesmal das Serum in konstanter Menge $(0,1)$, das Antigen aber in fallenden Dosen zugesetzt. Die Versuche wurden stets mit demselben hämolytischen System, den doppelten Mengen der Titer-Dosis des hämolytischen Serums und unter absolut gleichen Versuchsbedingungen angestellt. Die Komplementverankerung wurde durch Untersuchung der Röhrchen eine Stunde nach $\mathrm{Zu}$ satz des hämolytischen Systems festgestellt und, nachdem die Röhrchen noch 24 Stunden bei Zimmertemperatur gestanden

1) Neuleld und $\mathrm{Handel}$, Arbeiten a us dem Kaiserlichen Gesundheitsamt. 2) Händel, Deutsche medizinische Wuchenschrift 1907, No. 49 
hatten, kontrolliert. Das Ergebnis der zahlreichen, oft wiederholten Versuche ergab nun, dab ein Unterschied im Titer bei Benutzung der verschiedenen Methoden nicht zutage trat. Im Prinzip sind die alte Methode Bordet-Gengou, bei welcher die Bakterien nicht abzentrifugiert werden, sowie die Modifikation derselben mit Abzentrifugierung und endlich die Methode, bei welcher das Antigen in Form von Extrakten benutzt wird, einander durchaus gleichwertig. Auch die Spezifizität der Reaktion und die Titerbestimmung der Sera ließ sich bei Benutzung von formerhaltenen Bakterien durchaus sicher feststellen. Wir brauchen deshalb für die Feststellung spezifischer, komplementverankernder Stoffe bei Meningococcenserum und Meningococcen nicht Extrakte heranzuziehen. Das ist von wesentlichem Vorteil für die Identifizierung von verdächtigen Kulturen mittels der Komplementverankerungsmethode. Es ist dadurch eine wesentliche Vereinfachung gegeben, die um so mehr praktische Bedeutung hat, als gerade bei dieser Bakterienart die Agglutination für die Identifizierung von verdächtigen Kulturen zuweilen nicht in Frage kommen kann. Denn manche solcher verdächtigen Kulturen zeigen, wie auch manche Meningococcenstämme, Spontanagglutination und sind deshalb ungeeignet für die Prüfung mittels dieser Methodik. In solchen zweifelhaften Fällen wird die Komplementverankerungsmethode nach BordetGengou in der ursprünglichen Form oder mit nachfolgender Zentrifugierung von praktischem Werte sein.

Nachdem durch diese Vorversuche eine völlige Klarheit über den Wert der einzelnen Methoden der Komplementverankerung für die Wertbestimmung des Meningococcenserums erzielt war, wurde die Wirkung des polyvalenten Meningococcenserums gegenüber verschiedenen Stämmen näher untersucht. Die Versuche wurden einerseits mit einem Meningococcenserum und dem homologen, zur Immunisierung des betreffenden serumspendenden Tieres benutzten Stamme angestellt. Zur Kontrolle wurde anderseits dasselbe Serum mit heterologen Stämmen, d. h. solchen, welche nicht zur Immunisierung der betreffenden Tiere gedient hatten, benutzt. Wenn die Titerwerte bei Benutzung ein und desselben Serums mit denselben Kulturen stets konstant sind, so treten bei Benutzung verschiedener Serumarten gegenüber ein und derselben Kultur und umgekehrt bei Verwendung verschiedener Kulturen gegenüber ein und demselben Serum Unterschiede zutage. Wie aus der folgenden Tabelle ersichtlich ist, werden nun häufig Stämme, die nicht zur Immunisierung benutzt waren, viel stärker beeinflußt als die homologen, zur Herstellung des Serums benutzten Kulturen. Diese Tatsache konnte nicht nur mit einem einzigen Serum, sondern mit verschiedenen Serumpräparaten, welche der Prüfung unterzogen wurden, festgestellt werden. Unterschiede zwischen den verschiedenen Methoden bestanden hierbei nicht; namentlich wurden bei Benutzung von Extrakten und Vollbakterien stets kongruente Resultate erhalten.

\section{Tabelle III.}

Endtitertabelle der Komplementverankerungsversuche mit Berner Serum und einer Reihe Meningococcenstämme.

Methode Neufeld: Verwendetes Kulturmaterial: $1 / 5$ Oese. Titer des hämolytischen Serums: 0,05. Hammelblutkörperchen: Frisch, zweimal gewaschen und zentrifugiert. Komplement: Frisches Meerschweinchenserum 0,05. Kochsalzlösung: Isotonisch $(0,85 \%)$. Meningococcenserum: Inaktiviert 1 Stunde bei $58-60^{\circ} \mathrm{C}$. In fallenden Dosen: $0,07,0,04,0,01,0,007,0,004,0,001$.

\begin{tabular}{|c|c|c|c|c|c|}
\hline & Verwendeter Stamm & \begin{tabular}{|c|} 
Endtiter mit \\
kompletter \\
Hemmung
\end{tabular} & & Verwendeter Stamm & $\begin{array}{l}\text { Endtiter mit } \\
\text { kompletter } \\
\text { Hemmung }\end{array}$ \\
\hline 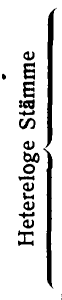 & $\begin{array}{l}\text { Berlin } 138 \text { neu } \\
\text { Metz Kr. } \\
\text { Metz G. } 1 \text { (Gerken) } \\
\text { Berlin Laz. I } \\
\text { Berlin-Beuthen neu } \\
\text { Frankfurt I. } \\
\text { Frankfurt III. } \\
\text { Höchst W. } \\
\text { Hochst G. } \\
\text { Gelsenkirchen } 1 . \\
\text { Gelsenkirchen } 239 \\
\text { Gelsenkirchen } 1040 \\
\text { Schlapka } \\
\text { Orleans }\end{array}$ & $\begin{array}{l}0,004 \\
0,07 \\
0,004 \\
0,004 \\
0,004 \\
0,07 \\
0,004 \\
0,004 \\
0,004 \\
0,01 \\
0,07 \\
0,007 \\
0,004 \\
0,004\end{array}$ & 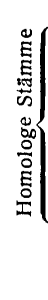 & $\begin{array}{l}\text { Riggisberg } \\
\text { Moutier } \\
\text { Beuthen } \\
\text { Biel } \\
\text { Berlin alt } \\
\text { Sahli } \\
\text { Worb } \\
\text { Meningococcus III. } \\
\text { Schwarzenburg } \\
\text { Solothurn } \\
\text { Mrosigk }\end{array}$ & $\begin{array}{l}0,007 \\
0,007 \\
0,004 \\
0,007 \\
0,004 \\
0,007 \\
0,004 \\
0,007 \\
0,01 \\
0,01 \\
0,007\end{array}$ \\
\hline
\end{tabular}

Der Gehalt des Serums an Agglutininen und der Gehalt an Stoffen, welche eine Komplementbindung mit Bakterien verursachen, weisen dabei oft grobe Unterschiede auf. Am auffälligsten war dieses Verhalten bei dem uns durch die Liebenswürdigkeit von Prof. Ruppel aus den Laboratorien der Höchster Farbwerke zur Verfügung gestellten Meningococcenserum. Dieses Serum besab stark agglutinierende Eigenschaften, wie aus der folgenden Tabelle ersichtlich ist. Bei Benutzung der Komplementverankerungsmethode konnten aber nur Spuren spezifischer Stoffe nachgewiesen werden. Es wurden die ver. schiedensten Methoden angewandt und eine ganze Anzahl von Meningococcenkulturen zur Prüfung des Höchster Serums herangezogen, immer mit dem gleichen Erfolg. Es wird durch diese Tatsache auch aufs deutlichste demonstriert, daß die Komplementverankerung mit der Agglutination nicht das geringste zu tun hat. Nach den Angaben von Ruppel soll das Serum einen hohen Schutzwert gegenüber der von ihm benutzten hochvirulenten Kultur im Mäuseversuch zeigen.

Tabelle IV.

Vergleichstabelle der Agglutination und Komplementverankerung mit Höchster Serum.

Verankerung:

Agglutination.

Fallende Serumdosen, Kulturmaterialkonstante $=1 / 5$ Oese. Hämol. Titer

Stämme: 1. Beuthen; $=0,003$. Beobachtung nach $1 \mathrm{u} .24$ 2. Biel; 3. Berlin. Stunden. Alte Methode.

\begin{tabular}{|c|c|c|c|c|c|c|c|c|c|c|c|c|c|c|}
\hline & $\stackrel{8}{=}$ & ‡ & $\stackrel{8}{=}$ & $\stackrel{8}{-}$ & 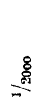 & $\frac{8}{80}$ & $\stackrel{8}{\frac{8}{-1}}$ & 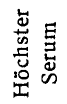 & 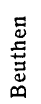 & $\bar{\oplus}$ & 㤩 & 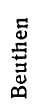 & $\stackrel{\bar{\Xi}}{\ddot{\varphi}}$ & 䓌 \\
\hline $\begin{array}{l}1 . \\
2 . \\
3 .\end{array}$ & $\stackrel{+}{+}+$ & + & $\stackrel{+}{+}$ & $\stackrel{+}{+}$ & $\stackrel{+}{+}+$ & $\begin{array}{r}0 \\
+ \\
+\end{array}$ & $\begin{array}{c}0 \\
0 \\
+\end{array}$ & $\begin{array}{l}0,4 \\
0,1 \\
0,07 \\
0,04 \\
0,01\end{array}$ & $\begin{array}{c} \pm \\
0 \\
0 \\
0 \\
0\end{array}$ & $\begin{array}{l} \pm \\
0 \\
0 \\
0 \\
0\end{array}$ & $\begin{array}{l} \pm \\
0 \\
0 \\
0 \\
0\end{array}$ & $\begin{array}{l} \pm \\
.0 \\
0 \\
0 \\
0\end{array}$ & $\begin{array}{l} \pm \\
.0 \\
0 \\
0 \\
0\end{array}$ & $\begin{array}{l} \pm \\
.0 \\
0 \\
0 \\
0\end{array}$ \\
\hline
\end{tabular}

Da an der Tatsächlichkeit dieser Angaben wohl kaum zu zweifeln ist, so würde daraus hervorgehen, daß die Komplementverankerung nicht ohne weiteres den Gehalt an bakteriolytischen Schutzstoffen, wie sie durch den Pfeifferschen Versuch festgestellt werden, (Ambozeptoren) anzeigt.

Es wurden nun ferner Versuche angestellt, um zu untersuchen, wie lange die spezifischen, verankernden Stoffe einerseits in den flüssigen Serumpräparaten, anderseits in trockenem Serum haltbar sind, d. h. sich mittels der von uns befolgten Methodik nachweisen lassen. Die Versuche ergaben, daß der Titer flüssiger, karbolisierter Sera verhältnismäßig rasch heruntergeht. Die Abnahme des Agglutinationstiters geht dieser Abnahme des Gehaltes an Immunitätseinheiten nicht parallel. Auch im trockenen Meningococcenserum erfolgt eine Abnahme der spezifischen, durch diese Methode nachweisbaren Stoffe, wenn auch erheblich langsamer als im flüssigen. Aber selbst im Serum, welches neun Monate und länger konserviert ist, lassen sich immer noch komplementverankernde Stoffe ebenso wie spezifische Agglutinine nachweisen. Die Ambozeptoren des Cholera- und Typhusserums sind im Gegensatz zu den komplementbindenden Stoffen des Meningococcenserums sehr lange haltbar, ohne wesentliche Einbuße an Wirksamkeit zu erfahren. Auch diese Tatsache spricht dafür, daß die komplementbindenden Stoffe keine echten Ambozeptoren, wie sie beim Pfeifforschen Phänomen in Wirksamkeit treten, sind. Es ergibt sich hieraus jedenfalls die praktische Folgerung, daß das Meningococcenserum nicht allzulange aufbewahrt werden darf, weder das für diagnostische, wissenschaftliche $Z$ wecke $\mathrm{zu}$ verwendende noch das therapeutisch zu benutzende. Es sollte vielmehr für die therapeutische Behandlung des meningitiskranken Menschen Serum, welches älter als drei Monate ist, wenn möglich, nicht verwendet werden.

Eine deutliche Schädigung erfahren die durch Komplementverankerung nachweisbaren Stoffe auch durch einstündige Erhitzung auf $60^{\circ} \mathrm{C}$. Während wir von echten Ambozeptoren wissen, daß eine derartige Erhitzung sie garnicht schädigt, nimmt der Titer des erhitzten Meningococcenserums an Komplementverankerungsstoffen bei Benutzung der Bordet- 
Gengouschen Methode ganz erheblich ab. Es empfiehlt sich deshalb für vergleichende Untersuchungen, namentlich auch für Wertbestimmungen, das Komplement, welches ja in jedem Serum enthalten ist und bei den Komplementverankerungsversuchen störend für die Messung der Stoffe einwirkt, nicht durch Erwärmung, sondern einfach durch Konservierung des Serums zu entfernen. Wie unsere Versuche zeigen, ist nach acht Tagen auch nicht eine Spur von Komplement durch die BordetGengousche Versuchsanordnung im flüssig aufbewahrten Meningococcenserum nachzuweisen, selbst nicht bei Verwendung größerer Mengen von Meningococcenserum. Die Hämolyse tritt aber bei derartigen Versuchen sofort ein, wenn frisches Komplement dem Gemisch hinzugesetzt wird.

Im Gegensatz zur Labilität der spezifischen Stoffe des Meningococcenserums sind die Antigene relativ haltbar. Sterile Extrakte aus Meningococcenkulturen, gewonnen durch Schütteln mit destilliertem Wasser, erwiesen sich trotz Karbolzusatzes nach noun Monaten noch durchaus geeignet zum Versuch; allerdings tritt eine gewisse Verringerung der bindenden Kraft der Antigene ein, aber man kann sich doch auf diese Weise eine Testflüssigkeit herstellen, die für längere Zeit und gröBere Versuchsreihen ausreichend ist.

Die Bindungskraft von Meningococcenextrakten, die aus verschiedenen Kulturen hergestellt sind, entspricht im wesentlichen der Konzentration des Extraktes. Je konzentrierter das Extrakt ist, desto stärker die Bindungskraft, und umgekehrt. Benutzt man aber stets die gleiche Methode der Herstellung solcher Extrakte aus Kulturen und kontrolliert die Extrakte außerdem auf optischem Wege (Intensität der gelblichen Färbung), so läßt sich eine ziemliche Konstanz der Extrakte von vornherein garantieren. Zur genauen Einstellung ist es dann notwendig, mit Hilfe eines Standardserums den Titer exakt zu bestimmen.

Es ist von einigen Autoren behauptet worden, daß in der Bindungsfähigkeit junger und alter Meningococcenkulturen erhebliche Unterschiede beständen. Wir haben unter Benutzung der Versuchsanordnung mit Vollbakterien diese Behauptung einer genauen experimentellen Prüfung unterzogen. Es wurden zwölfstündige sowohl wie die mehrere Tage lang gewachsenen Meningococcenkulturen, von denen die ältesten elf Tage im Brutschrank aufbewahrt waren, zu den Versuchen benutzt. Es lieben sich auch nicht die geringsten Unterschiede in der Bindungsfähigkeit der Kulturen feststellen. Diese Tatsache konnte nicht nur bei Benutzung von homologen, sondern auch von heterologen Serumproben mehrfach bestätigt werden.

Eine Lockerung des Komplements haben wir bei Benutzung von Vollbakterien, wenn dieselben nicht abzentrifugiert wurden, nur in der Nähe der Grenzdosen mehrfach beobachtet. Diese Lockerung tritt allerdings erst nach 24 Stunden zutage. Es genügt aber vollkommen, die Titerbestimmung nach einer Stunde vorzunehmen. Innerhalb dieser Zeit haben wir aber niemals eine Loslösung bzw. Lockerung des Komplements beobachten können.

Zusammenfassung. Die Ergebnisse unserer Untersuchung, delun belege ausfürlich in den bei Gustav Fischer in Jena erscheinenden Arbeiten aus dem Institut zur Erforschung der Infektionskrankheiten mitgeteilt werden sollen, lauten : Entgegen den Angaben von Kraus und Dörr ${ }^{1}$ ) sowie von Ballner und Reibmeyer ${ }^{2}$ ) bildet die Methode der Komplementverankerung eine Bereicherung der serumdiagnostischen Methoden und kann zur Wertbestimmung des Meningococcenserums benutzt werden. Die Methode gibt in der Hand des Geübten, wenn alle Kontrollversuche, wie wir es stets getan haben, herangezogen werden, durchaus gleichmäßige und auBerordentlich konstante Resultate. Wenn man nur genau quantitativ arbeitet, so ist es gleichgültig, ob man Extrakte von Bakterien oder die formerhaltenen Meningococccen selbst für die Komplementverankerung benutzt.

Wenn auch durch diese Untersuchungen mit Meningococcen die Frage nicht sicher entschieden werden kann, ob die Komplementverankerungsmethode einen Nachweis von bakteriolytischen Ambozeptoren gestattet, so deuten die Ergebnisse der Versuche, namentlich die Thermo-Labilität und geringe Haltbarkeit der ablenkenden Stoffe sowie das Fehlen derselben in dem in den Höchster Farbwerken hergestellten agglutinierenden und nach Ruppels Angaben Schutz- und Heilwirkung entfaltenden Serum doch darauf hin, daß die durch Komplementverankerung nachweisbaren Stoffe keine echten Ambozeptoren, sondern höchstwahrscheinlich spezifische Stoffe eigener Art sind. Es würde also die von Neufeld und Hüne,1) Händel ${ }^{2}$ ) sowie Moreschis ${ }^{3}$ für das Typhus- und Choleraserum nachgewiesene Inkongruenz des Gehaltes an Ambozeptoren und verankernden Stoffen auch durch unsere Untersuchungen über Meningococcenserum Bestätigung erfahren. Die komplementverankernden Stoffe des Meningococcenserums gehen nach unseren Untersuchungen auch der Agglutination nicht parallel. Diese Frage. kollidiert aber garnicht mit der Tatsache, daß die Methode der Komplementverankerung nach Bordet-Gengou für die Wertbestimmung des Meningococcenserums so lange wertvolle Dienste leistet, bis wir mit Sicherheit tierpathogene Stämme herstellen oder lösliche Meningococcengifte gewinnen können. Beides bietet aber bisher wenig Aussicht auf Erfolg, wenn sich nicht die Angaben von Kraus und Dörr über die aus Agarkulturen hergestellten Gifte bewahrheiten.

Die durch Komplementverankerung nachweisbaren Stoffe sind durchaus spezifisch. Sie sind auch für die Differenzierung und Identifizierung der Meningococcen praktisch brauchbar. Selbst in Bakteriengemischen läßt sich mittels dieser Methode der Identitätsnachweis für formerhaltene oder gelöste Meningococcen erbringen, sie zeigt sich also hier überlegen der Agglutinationsreaktion. Prüft man eine größere Anzahl von Meningococcenkulturen unter gleichen Bedingungen mit ein und demselben Serum, so zeigen sich ganz erhebliche Unterschiede in den Grenzwerten. Dieselben treten auch bei polyvalenten Serumpräparaten auf und sind in letzterem Falle auch bei den einzelnen zur Herstellung des polyvalenten Serums benutzten Kulturen nachweisbar. Sie gehen nicht parallel mit den durch Agglutinationsprüfung erhaltenen Werten. Bei Benutzung einer und derselben Kultur bzw. ihrer Extrakte läßt sich der Gehalt an komplementverankernden Stoffen verschiedener Sera feststellen. Es konnte die bemerkenswerte Tatsache ermittelt werden, daß das in den Höchster Farbwerken hergestellte Serum nach Ruppel eine ausgesprochene Schutzkraft hat, da die von $R$ up pel benutzten Stämme für Mäuse eine Dosis letalis von $\frac{1}{100000}$ Oese bei intraperitonealer Injektion besaßen, aber trotz hoher Agglutinationskraft fast keine spezifischen Stoffe mittels der Bordet-Gengou schen Methode erkennen ließ. Eine geringe Bindungsfähigkeit zeigte auch das von der Firma Merck hergestellte Serum trotz hoher Agglutinationskraft. Dagegen ließen sich bei den in dem Berliner bzw. Berner Institut zur Erforschung der Infektionskrankheiten hergestellten Serumpräparaten nicht nur annähernd gleiche Agglutinationstitres, sondern auch ein gleich hoher Gehalt an spezifischen Stoffen durch die Komplementverankerung nachweisen.

1) Neufeld und $H$ ũ ne, Arbeit aus dem Kaiserlichen Gesundheitsamt Bd. 25. 2) Hande1, Deutsche medizinische Wochenschrift 1907, Dezember. - 3) Moreschi, Berliner klinische Wochenschrift 1907 . 